\title{
Natural Deduction for Diagonal Operators
}

Fabio Lampert

Draft of May 2017. Forthcoming in Proceedings of the Canadian Society for

History and Philosophy of Mathematics.

\begin{abstract}
We present a sound and complete Fitch-style natural deduction system for an $\mathbf{S 5}$ modal logic containing an actuality operator, a diagonal necessity operator, and a diagonal possibility operator. The logic is two-dimensional, where we evaluate sentences with respect to both an actual world (first dimension) and a world of evaluation (second dimension). The diagonal necessity operator behaves as a quantifier over every point on the diagonal between actual worlds and worlds of evaluation, while the diagonal possibility quantifies over some point on the diagonal. Thus, they are just like the epistemic operators for apriority and its dual. We take this extension of Fitch's familiar derivation system to be a very natural one, since the new rules and labeled lines hereby introduced preserve the structure of Fitch's own rules for the modal case.
\end{abstract}

\section{Introduction}

One familiar doctrine in contemporary analytic philosophy says, roughly, that a sentence is a priori knowable if and only if it is true along the diagonal. Leaving aside several important qualifications and distinctions, this characterization of the a priori and others very similar to it have appeared in Stalnaker (1978), Evans (1979), Davies and Humberstone (1980), Kaplan (1989), Jackson (1998), Chalmers (1996, 2004), and others. These constitute the core of the research program identified as two-dimensional semantics 1 In general, two-dimensional semanticists defend an extension of the formal apparatus of possible worlds semantics in order to analyze the meaning of linguistic expressions. They claim that a sentence should be evaluated not only with respect to a single index or possible world, but to a pair thereof,

Fabio Lampert

University of California, Davis, USA, e-mail: fabiodcleyahoo.com.br

${ }^{1}$ If we can say that these constitute a single research program at all. In any case, Chalmers (2004) provides a nice account of how exactly most of these differ. 
whereby different kinds of two-dimensional semantics will then claim distinct interpretations for the new indices. To say that a sentence is a priori knowable then comes down to it being true at every pair of indices where the first and second coordinate are identical, that is, the diagonal between pairs of indices/possible worlds (see Figure 1).

$$
\left(\begin{array}{cccccc} 
& w_{0} & w_{1} & w_{2} & w_{3} & \cdots \\
w_{0} & \varphi & & & & \\
w_{1} & & \varphi & & & \\
w_{2} & & & \varphi & & \\
w_{3} & & & & \varphi & \\
\vdots & & & & & \ddots
\end{array}\right)
$$

Fig. 1 A 2D-matrix for a diagonally true sentence $\varphi$.

Extant presentations of the formalism involved in several versions of twodimensionalisms have been either semantic or, when proof theoretic, axiomatic in character $2^{2}$ For example, Davies and Humberstone (1980) present an axiomatization of a two-dimensional system that arguably captures some important a priori truths as a formal rendering of Evans' (1979) notion of deep necessity ${ }_{3}^{3}$ Evans distinguished between superficial and deep necessity in order to account for Kripke's (1980) examples of the contingent a priori. For Evans, superficial necessity or contingency is a matter of how a sentence behaves when embedded within the modal operators $\square$ and $\diamond$; by contrast, deep necessity and contingency have to do with the necessity or contingency of the content expressed by the sentence in question. By stipulating that 'Julius', for instance, names the inventor of the zip, the sentence (S) 'if anyone uniquely invented the zip, Julius invented the zip' is deeply necessary, for by understanding what is expressed by (S) we know it merely says that if anyone uniquely invented the zip, it was the inventor of the zip himself. And this, i.e. what is expressed by (S), is knowable a priori. Nonetheless, (S) is superficially contingent, for it is easily seen to be false at every possible world where the inventor of the zip differs from William Whitworth - the actual inventor of the zip. Davies and Humberstone's idea was to formalize these two notions of necessity (contingency) by adding to a modal language their 'fixedly actually' operator (see fn. 3 ) alongside the usual modal operators $\square$ and $\diamond$. In their system, formulas are evaluated with respect to pairs of possible worlds, whence the logic is said to be two-dimensional.

\footnotetext{
2 The exception is Restall (2012), as mentioned below.

${ }^{3}$ Thereby relegating $\square$ to superficial necessity. Strictly speaking, the axiomatic system in Davies and Humberstone (1980) does not contain a diagonal operator, but a 'fixedly' operator, $\mathscr{F}$, which alongside an actuality operator, $\mathscr{A}$, generates truth on the diagonal. It is with respect to $\mathscr{F} \mathscr{A}$ that deep necessity is defined. For more on the notions of deep and superficial necessity see Evans (1979).
} 
Each pair is constituted by a possible world and a world that is considered (intuitively) as the actual one. Thus, a sentence $\varphi$ is fixedly actually true just in case it holds along the diagonal or, in other words, just in case $\varphi$ is true at every possible world that is considered as the actual one ${ }^{4}$ Since the examples that are relevant to the distinction made by Evans between superficial and deep necessity (contingency) can be formalized within their two-dimensional system, Davies and Humberstone claimed to have a logic in which these two kinds of modalities are represented.

In addition to Davies and Humberstone's axiomatic system, there is a hypersequent system in Restall (2012) and an (equivalent) axiomatic system in Fritz (2013, 2014) designed to capture some of the core formal aspects of versions of twodimensional semantics. These systems involve the usual modal operators, a primitive diagonal (apriority) operator, and an actuality operator. In particular, Fritz's system is motivated by the epistemic two-dimensionalism developed by Chalmers, where the indices of evaluation are intuitively interpreted as pairs constituted by epistemic scenarios and possible worlds, or epistemic and metaphysical possibilities, respectively. Since there is an epistemic possibility in which water is not $\mathrm{H}_{2} \mathrm{O}$ (i.e. the XYZ-possibility), the sentence ( $\mathrm{S}^{*}$ ) 'water is $\mathrm{H}_{2} \mathrm{O}$ ' is not a priori knowable, although it is necessary. More formally, we can say that there is a point along the diagonal where $\left(\mathrm{S}^{*}\right)$ is false, but no point along the horizontal where $\left(\mathrm{S}^{*}\right)$ is false (see, again, Figure 1). In contrast, there is no epistemic possibility where ( $\left.\mathrm{S}^{* *}\right)$ 'water is water' fails to hold, whence $\left(\mathrm{S}^{* *}\right)$ holds at every scenario-world pair along the diagonal. Thus, a sentence is a priori just in case it holds along the diagonal, and a posteriori otherwise.

The aim of this paper is to present a Natural Deduction system that exactly captures the behaviour of the diagonal operator and its interaction with the necessity operator in an $\mathbf{S 5}$ modal logic with an added actuality operator, $\left.\mathscr{A}\right|^{5}$ Modal systems containing $\mathscr{A}$ are by no means novelty. Some representatives are found as early as in Crossley and Humberstone (1977) — an axiomatic system — and Hazen (1978) - a natural deduction system. However, we know of no natural deduction system for two-dimensional modal logics. One of the virtues of the present system is that it is extremely easy to use for anyone already familiarized with traditional Fitch-style natural deduction systems for modal logics, thereby making the logical properties of the diagonal modalities, and some of the core ideas of two-dimensional semantics, transparent to a wider range of readers, in contrast with the (usually more cumbersome) axiomatic treatments ${ }^{6}$

\footnotetext{
${ }^{4}$ Note that (S) holds at every world considered as actual, since in these worlds 'Julius' always picks out the actual inventor of the zip. If, by contrast, the actual world is held fixed, and we only consider different possible worlds relative to this world as the actual one, then there will be pairs at which (S) is false - precisely those in which the inventor of the zip differs from the actual one.

${ }^{5}$ We purposefully conflate use/mention when the context is clear to avoid cluttering the paper.

6 In Lampert (forthcoming) we present several prefixed tableau systems for different twodimensional modal logics.
} 


\section{The system $\mathrm{S5E}_{2 D}$}

Definition 1 (Basic Language). Let $p, q, r \ldots$ be a countable list of members of the set $A T$ of propositional letters. The language $\mathscr{L}_{\mathscr{A} \mathscr{D}}$ is defined recursively by the following grammar:

$$
\varphi::=A T|\neg \varphi|(\varphi \wedge \psi)|\square \varphi| \mathscr{A} \varphi \mid \mathscr{D} \varphi
$$

The other Boolean connectives are defined as usual, and we also define the duals $\diamond:=\neg \square \neg$ and $\mathscr{C}:=\neg \mathscr{D} \neg$.

Definition 2 (2D-model). A $2 D$-model is a tuple, $\mathscr{M}=\left\langle W, \mathscr{R}_{\square}, \mathscr{R}_{\mathscr{D}}, V\right\rangle$, where

1. $W=Z \times Z$ for some set $Z$,

2. $\mathscr{R}_{\square} \subseteq W \times W$, the $\square$-accessibility relation, is the least relation such that for every $v, w, z \in Z,\langle w, v\rangle \mathscr{R}_{\square}\langle z, v\rangle$,

3. $\mathscr{R}_{\mathscr{D}} \subseteq W \times W$, the $\mathscr{D}$-accessibility relation, is the least relation such that for every $v, w, z \in Z,\langle w, v\rangle \mathscr{R}_{\mathscr{D}}\langle z, z\rangle$, and

4. $V$ is a function assigning sets $V(p)$ of pairs of possible worlds to each member of $A T$.

Definition 3 (Truth). We define ' $\varphi$ is true at $w$ with respect to $v$ in $\mathscr{M}$ ', written $\mathscr{M},\langle w, v\rangle \vDash \varphi$, by recursion on $\varphi$. Here $w$ is the typical world of evaluation while $v$ is the actual world under consideration. Intuitively, $v$ might also be taken as an epistemic scenario or possibility. For a pair $\langle w, v\rangle \in W$,

$$
\begin{aligned}
& \mathscr{M},\langle w, v\rangle \vDash p \Longleftrightarrow\langle w, v\rangle \in V(p) ; \\
& \mathscr{M},\langle w, v\rangle \vDash \neg \varphi \quad \Longleftrightarrow \mathscr{M},\langle w, v\rangle \not \models \varphi \\
& \mathscr{M},\langle w, v\rangle \vDash(\varphi \wedge \psi) \Longleftrightarrow \mathscr{M},\langle w, v\rangle \vDash \varphi \text { and } \mathscr{M},\langle w, v\rangle \vDash \psi ; \\
& \mathscr{M},\langle w, v\rangle \vDash \diamond \varphi \Longleftrightarrow \text { for some } z \in Z,\langle w, v\rangle \mathscr{R}_{\square}\langle z, v\rangle \text { and } \mathscr{M},\langle z, v\rangle \vDash \varphi ; \\
& \mathscr{M},\langle w, v\rangle \vDash \square \varphi \quad \text { for every } z \in Z \text { such that }\langle w, v\rangle \mathscr{R}_{\square}\langle z, v\rangle, \mathscr{M},\langle z, v\rangle \vDash \varphi ; \\
& \mathscr{M},\langle w, v\rangle \vDash \mathscr{A} \varphi \quad \Longleftrightarrow \mathscr{M},\langle v, v\rangle \vDash \varphi ; \\
& \mathscr{M},\langle w, v\rangle \vDash \mathscr{C} \varphi \quad \Longleftrightarrow \text { for some } z \in Z,\langle w, v\rangle \mathscr{R}_{\mathscr{D}}\langle z, z\rangle \text { and } \mathscr{M},\langle z, z\rangle \vDash \varphi . \\
& \mathscr{M},\langle w, v\rangle \vDash \mathscr{D} \varphi \Longleftrightarrow \text { for every } z \in Z \text { such that }\langle w, v\rangle \mathscr{R}_{\mathscr{D}}\langle z, z\rangle, \mathscr{M},\langle z, z\rangle \vDash \varphi .
\end{aligned}
$$

Definition 4 (Truth, validity, and consequence). A sentence, $\varphi$, is true in a $2 D$ model $\mathscr{M}=\left\langle W, \mathscr{R}_{\square}, \mathscr{R}_{\mathscr{D}}, V\right\rangle$, written $\mathscr{M} \vDash \varphi$, if and only if $\mathscr{M},\langle w, w\rangle \vDash \varphi$ for every $\langle w, w\rangle \in W$; a sentence $\varphi$ is diagonally valid in a class $\mathscr{C}$ of models if and only if it is true in every model in $\mathscr{C}$; and a sentence $\varphi$ is a logical consequence of a set of sentences $\Gamma$ if and only if for all models $\mathscr{M}=\left\langle W, \mathscr{R}_{\square}, \mathscr{R}_{\mathscr{D}}, V\right\rangle$ and $\langle w, w\rangle \in W$, if $\mathscr{M},\langle w, w\rangle \vDash \gamma$ for all $\gamma \in \Gamma$, then $\mathscr{M},\langle w, w\rangle \vDash \varphi$. 


\section{Natural Deduction System for $\mathbf{S 5 E}_{2 D}$}

In Fitch's original presentation for modal logic a natural deduction system is enriched with $\square$-labeled lines to denote the introduction of a possible world in a derivation. In other words, $\square$-labeled lines are world-tags, for arbitrary possible worlds. This means that the formulas occurring in a derivation within their scope should be evaluated at the possible world introduced by the respective $\square$-labeled line. The new subproofs introduced by $\square$-labeled lines are called strict or just $\square$-subproofs. In one-dimensional modal logic we use $\square$-labeled lines in cases where we need to eliminate or introduce the modal operators $\square$ and $\diamond$. For instance, we can prove $\diamond p \supset \square \diamond p$ in $\mathbf{S 5}$ by the following derivation:

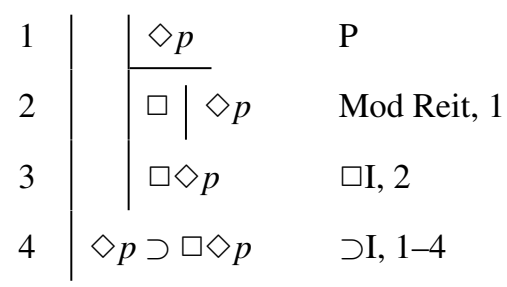

By contrast, if we want to evaluate formulas with respect to a pair of worlds, as our two-dimensional framework requires, we should expect the system to be a bit more complicated, for our Fitch-lines must dictate that the formulas are true at a world with respect to a certain world considered as actual, or simply that the formula is true at a pair of possible worlds. Thus, there is a further dimension present in what our labeled lines ought to convey in the proof system. Our Fitch-lines, as a result, will comprise a $\square$-labeled line, an actuality labeled line denoted by $\mathscr{A}$, and a diagonal labeled line denoted by $\delta$, the latter introduced specifically for diagonal operators.

In what follows we show the Fitch rules necessary for $\mathbf{S 5} \mathrm{E}_{2 D}$. For ease of presentation, rather than listing all of them at once, first we recall the rules for $\mathbf{S 5}$, then we show the modifications needed to accommodate the actuality operator, and only after that we add the rules for the entire two-dimensional system. Thus, in effect, we present natural deduction systems for three different logics ordered by inclusion: $\mathrm{S5}, \mathbf{S 5} \mathscr{A}$, and $\mathrm{S5E}_{2 D}$.

\section{$3.1 S 5$}

In S5 the rules for the Boolean connectives are just the standard Fitch rules for propositional logic that one finds in any logic textbook. The rules governing the modal operators are the following:

( $\square$ I) $\square$ Introduction 


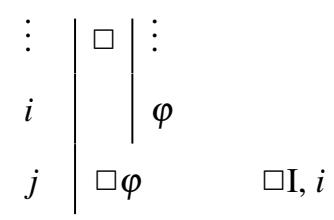

( $\square$ E) $\square$ Elimination

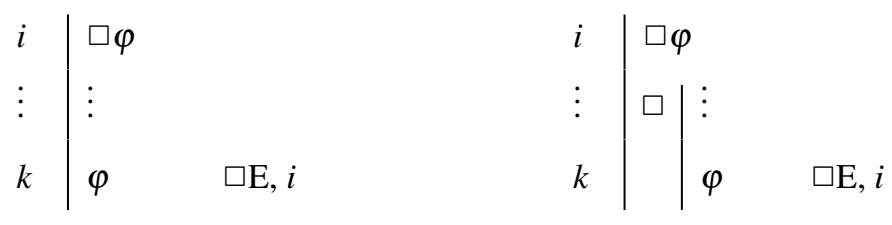

$(\diamond \mathrm{I}) \quad \diamond$ Introduction

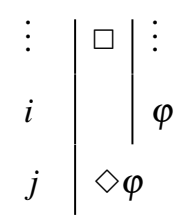

$\diamond \mathrm{I}, i$

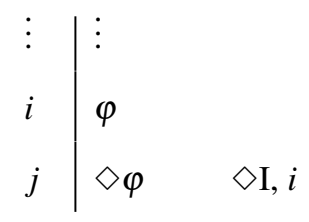

$(\diamond \mathrm{E}) \diamond$ Elimination

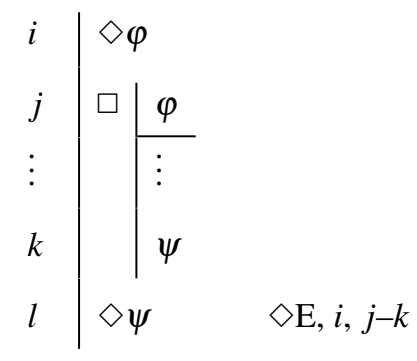

(MN) Modal Negation
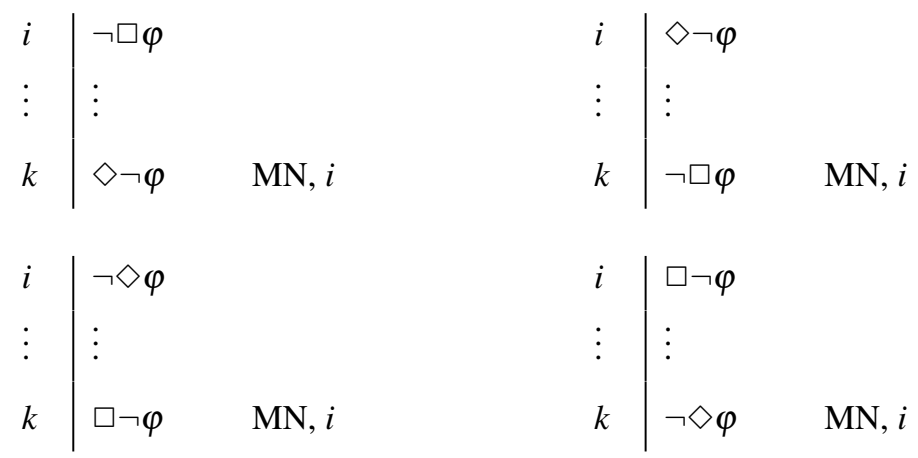


\section{$3.2 S 5 \mathscr{A}$}

The derivation system for the actuality operator is essentially the same as the one in Hazen (1978). Let an $\mathscr{A}$-labeled line denote a new kind of strict subproof, which we might also call $\mathscr{A}$-subproof. Moreover, we now say that the main proof is also counted as an $\mathscr{A}$-subproof. Now add the following rules to the ones presented above for $\mathbf{S 5}$ :

$(\mathscr{A}$ I) Actuality Introduction

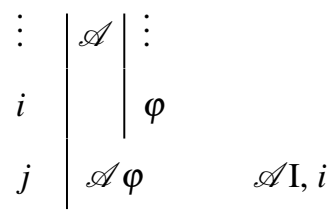

(act-I) act-I (only in $\mathscr{A}$-subproofs)

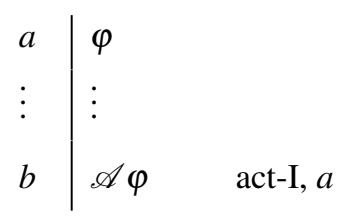

$(\mathrm{N} \mathscr{A})$ Negated Actuality

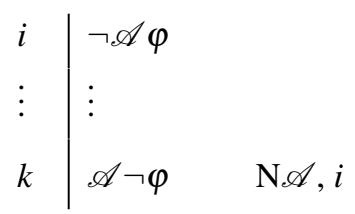

$(\mathscr{A}$ E) Actuality Elimination

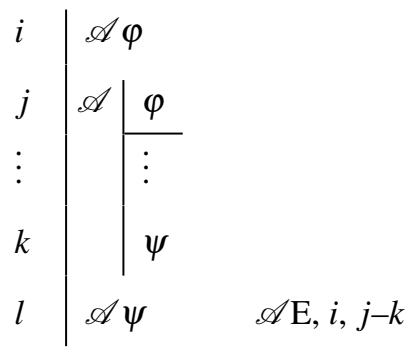

(act-E) act-E (only in $\mathscr{A}$-subproofs)

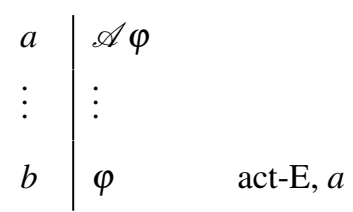

The rules of reiteration are essentially the same as for $\square$-subproofs, i.e. $\mathscr{A}$-formulas can be reiterated into $\square$-subproofs and vice-versa. Hazen (1978, especially pp. 618619) sketches the completeness theorem for the system $\mathbf{S 5} \mathscr{A}$.

\section{$3.3 S_{5} E_{2 D}$}

For the system $\mathbf{S 5} \mathrm{E}_{2 D}$ we have all the rules presented above, besides small restrictions that we shall mention in due course. Next we introduce the rules for diagonal 
operators, which turn out to be very similar to the usual modal rules except for the addition of $\delta$-subproofs.

( $\mathscr{D})$ Diagonal Necessity Introduction

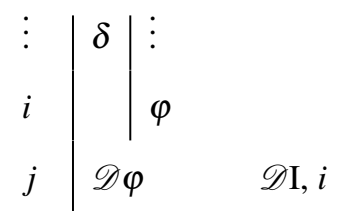

$(\mathscr{D E})$ Diagonal Necessity Elimination
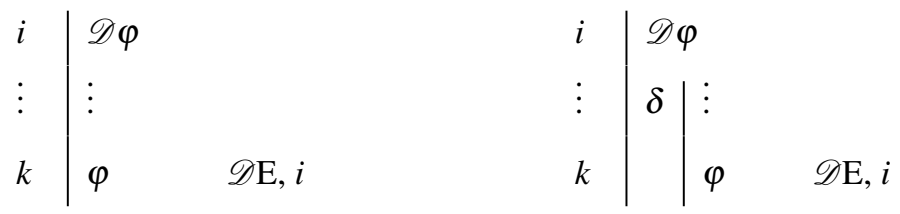

(CI) Diagonal Possibility Introduction

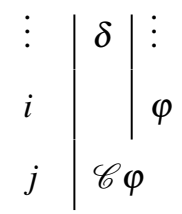

$\mathscr{C} \mathrm{I}, i$

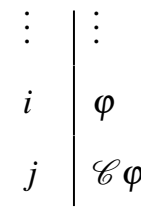

$\mathscr{C} \mathrm{I}, i$

(CEE) Diagonal Possibility Elimination

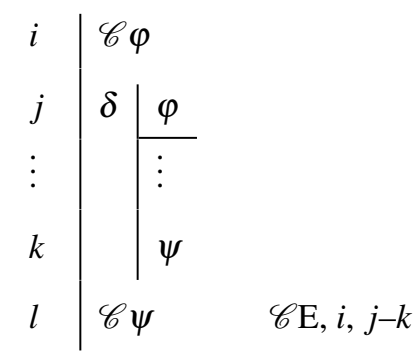

(DN) Diagonal Negation
\begin{tabular}{l|l}
$i$ & $\neg \mathscr{D} \varphi$ \\
$\vdots$ & $\vdots$ \\
$k$ & $\mathscr{C} \neg \varphi$
\end{tabular}
$\mathrm{DN}, i$
\begin{tabular}{l|l}
$i$ & $\mathscr{C} \neg \varphi$ \\
$\vdots$ & $\vdots$ \\
$k$ & $\neg \mathscr{D} \varphi$
\end{tabular}
$\mathrm{DN}, i$ 


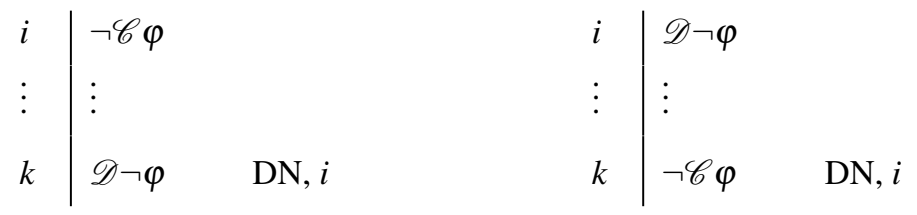

Concerning the restrictions mentioned above, the rules for the Boolean connectives remain the same, the only exception being the reductio ad absurdum rules, for we allow the relevant contradiction to occur within the scope of any $\square, \mathscr{A}$, or $\delta$-subproof. More importantly, $\square, \diamond$, and $\mathscr{A}$-formulas can still be reiterated within $\square$ and $\mathscr{A}$-subproofs, but they cannot be reiterated across $\delta$-subproofs. Thus, where $\# \in\{\square, \mathscr{A}\}$, and $\varphi$ is either a $\square, \diamond$, or $\mathscr{A}$-formula,

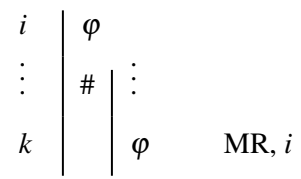

Fig. 2 Modal Reiteration Rule.

For example, the following instance of modal reiteration in the derivation on the left side is permissible, but the one on the right side is not:
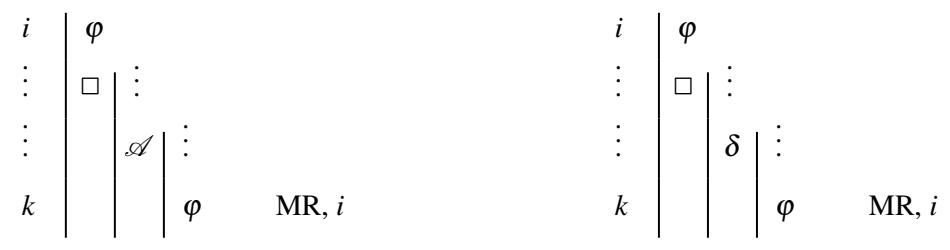

Fig. 3 Good and bad uses of modal reiteration.

The rule of diagonal reiteration, by contrast, can be used unrestrictedly in a derivation. Thus, in the derivation on the right side of Figure 3, the rule of diagonal reiteration (DR), rather than modal reiteration (MR), would allow the introduction of $\varphi$. More generally, where $\# \in\{\square, \mathscr{A}, \delta\}$, and $\varphi$ is any formula beginning with a diagonal operator, 


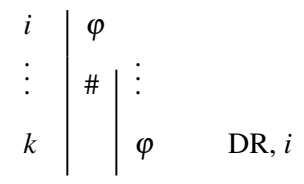

Fig. 4 Diagonal Reiteration Rule.

Finally, the (act-I) and (act-E) rules are also allowed within $\delta$-subproofs, while the other rules remain unchanged. In what follows we present some examples of derivations in $\mathbf{S 5 E}_{2 D}$, commenting (when necessary) on the usage of the rules just presented.

$$
\vdash \mathscr{D} p \supset \square \mathscr{D} p
$$

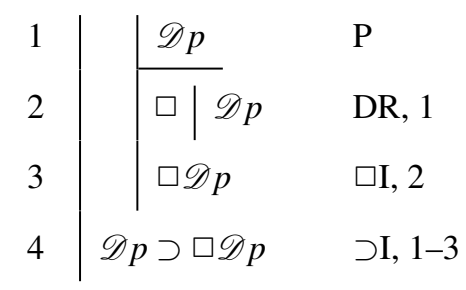

In this case, $\mathscr{D} p$ was legitimately reiterated over a $\square$-subproof since the reiteration rule for diagonal operators is unrestricted, while line 3 was obtained by usual application of $\square$ introduction. The following exemplifies a distinction between the two operators $\square$ and $\mathscr{D}$, especially in how they interact with the actuality operator:

$$
\begin{aligned}
& \vdash \mathscr{D}(\mathscr{A} p \supset p) \quad \nvdash \square(\mathscr{A} p \supset p)
\end{aligned}
$$

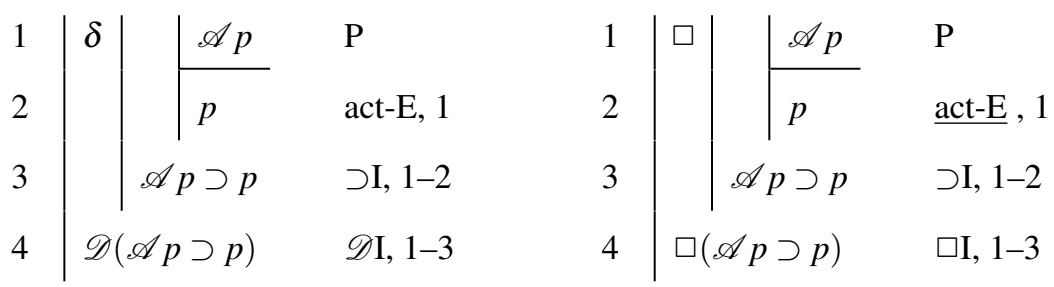

The problem with the derivation on the right is that (act-E) is not allowed unless it is applied within $\mathscr{A}$ and $\delta$-subproofs, as illustrated by the derivation on the left. In general, derivations containing only diagonal or the basic modal operators $\square$ and $\diamond$ will be analogous, but there are subtle differences in their interaction with $\mathscr{A}$. 


\section{Completeness for $\mathrm{S} \mathrm{E}_{2 D}$}

The derivation system defined above is clearly sound with respect to the twodimensional semantics presented in $\S 2$. Completeness, however, is not so obvious. We could establish it by a traditional Henkin-style argument extended to take care of pairs of possible worlds; however, it suffices to prove the axioms (by means of schematic derivations) of the system 2D presented in Fritz (2013). In effect, Fritz defines two systems, to wit, 2D and 2Dg. The former is based on a semantics just like the one defined in $\S 2$, whereas the latter characterizes truth in a model and the consequence relation in a general way, that is, with respect to every point in a $2 \mathrm{D}$ model: a sentence, $\varphi$, is generally true in a $2 D$-model if and only if $\mathscr{M},\langle w, v\rangle \vDash \varphi$ for every $\langle w, v\rangle \in W$; a sentence $\varphi$ is generally valid in a class $\mathscr{C}$ of models if and only if it is generally true in every model in $\mathscr{C}$; and a sentence $\varphi$ is a general logical consequence of a set of sentences $\Gamma$ if and only if for all models $\mathscr{M}=\left\langle W, \mathscr{R}_{\square}, \mathscr{R}_{\mathscr{D}}, V\right\rangle$ and $\langle w, v\rangle \in W$, if $\mathscr{M},\langle w, v\rangle \vDash \gamma$ for all $\gamma \in \Gamma$, then $\mathscr{M},\langle w, v\rangle \vDash \varphi$. While $\mathscr{A} \varphi \supset \varphi$ and $\mathscr{D} \varphi \supset \varphi$ are valid in $\mathbf{2 D}$, for instance, they are not valid in $\mathbf{2 D g}$, where truth, consequence, and validity are defined generally 7 But this distinction between general and diagonal validity can nonetheless be accommodated in our derivation system, whereby the latter is complete with respect to either notion of validity. The axioms 8 defining 2Dg comprise the following actuality base from Crossley and Humberstone's system $\mathbf{S 5} \mathscr{A} 9$, besides the usual $\mathbf{S 5}$ axioms:

$$
\begin{array}{ll}
(\mathscr{A} 1) \mathscr{A}(\mathscr{A} \varphi \supset \varphi) . & (\mathscr{A} 3) \mathscr{A} \varphi \equiv \neg \mathscr{A} \neg \varphi . \\
(\mathscr{A} 2) \mathscr{A}(\varphi \supset \psi) \supset(\mathscr{A} \varphi \supset \mathscr{A} \psi) .(\mathscr{A} 4) \mathscr{A} \varphi \supset \square \mathscr{A} \varphi .
\end{array}
$$

Moreover, the following axioms for diagonal operators are added by Fritz, with the (notational) difference that he uses $A$ rather than $\mathscr{D}$, and @ rather than $\mathscr{A}$ :

$$
\begin{aligned}
& \left(4_{\mathscr{D}}\right) \mathscr{D} \varphi \supset \mathscr{D} \mathscr{D} \varphi .\left(\mathbf{I}_{3}\right) \mathscr{D} \varphi \supset \mathscr{A} \varphi . \\
& \left(5_{\mathscr{D}}\right) \mathscr{C} \varphi \supset \mathscr{D} \mathscr{C} \varphi .\left(\mathbf{I}_{4}\right) \mathscr{D}(\mathscr{A} \varphi \supset \varphi) .
\end{aligned}
$$

If truth and validity are defined generally, as in $2 \mathrm{Dg}$, then the main proof of a derivation is not an $\mathscr{A}$-subproof anymore. (Note that this imposes a restriction on the derivation rules (act-I) and (act-E), while the other rules for the actuality operator remain sound.) Furthermore, $\mathscr{D}$ is not reflexive in $2 \mathrm{Dg}$, whence the reflexive rules for $(\mathscr{D} \mathrm{E})$ and $(\mathscr{C} \mathrm{I})$ must also be dropped in this case ${ }^{10}$ All the other rules are sound with respect to general validity, and the axioms above can be easily derived. On

\footnotetext{
${ }^{7}$ For a discussion on different accounts of validity in two-dimensional logics see Lampert (forthcoming).

${ }^{8}$ Strictly, axiom-schemata.

${ }^{9}$ Fritz does not list $\mathscr{A} 1$ between the axioms, but this appears in Crossley and Humberstone's original formulation (1977, p. 14).

10 These are the rules where no new scope line is opened in the elimination of $\mathscr{D}$ and the introduction of $\mathscr{C}$, respectively.
} 
the other hand, by defining truth and validity as in definition 2.4, we may add the following axioms to the ones above:

$$
(\mathscr{A} 5) \mathscr{A} \varphi \supset \varphi \cdot\left(\mathbf{I}_{T}\right) \mathscr{D} \varphi \supset \varphi .
$$

In fact, by just substituting $(\mathscr{A} 1)$ by $(\mathscr{A} 5)$ and adding $\left(\mathbf{I}_{T}\right)$ we get a complete axiomatization for 2D. The derivations of these two axioms in $\mathbf{S 5} \mathbf{E}_{2 D}$ are obvious and all the rules presented in $\S 3$ are sound with respect to diagonal validity.

In the following we exhibit schematic derivations for some of the axioms as illustration. The axioms governing the actuality operator are derived in Hazen (1978) in the midst of his proof that $\mathscr{A}$ is eliminable when added to the basic modal language. So we are left with the axioms for the diagonal operators, which we derive below. (A schematic derivation for $\mathbf{I}_{4}$ is just as illustrated in $\S 4$.)

$\vdash \mathscr{D} \varphi \supset \mathscr{D} \mathscr{D} \varphi$

\begin{tabular}{l|lll}
1 & $\mathscr{D} \varphi$ & $\mathrm{P}$ \\
2 & $\delta \mid \mathscr{D} \varphi$ & $\mathrm{DR}, 1$ \\
3 & & $\mathscr{D} \mathscr{D} \varphi$ & $\mathscr{D} \mathrm{I}, 2$ \\
4 & $\mathscr{D} \varphi \supset \mathscr{D} \mathscr{D} \varphi$ & $\supset \mathrm{I}, 1-3$
\end{tabular}

$\vdash \mathscr{C} \varphi \supset \mathscr{D} \mathscr{C} \varphi$

\begin{tabular}{l|lll}
1 & $\mathscr{C} \varphi$ & $\mathrm{P}$ \\
2 & $|\delta| \mathscr{C} \varphi$ & $\mathrm{DR}, 1$ \\
3 & & $\mathscr{D} \mathscr{C} \varphi$ & $\mathscr{D I}, 2$ \\
4 & $\mathscr{C} \varphi \supset \mathscr{D} \mathscr{C} \varphi$ & $\supset \mathrm{I}, 1-3$
\end{tabular}

$\vdash \mathscr{D} \varphi \supset \mathscr{A} \varphi$

\begin{tabular}{|c|c|c|}
\hline 1 & $\mathscr{D} \varphi$ & $\mathrm{P}$ \\
\hline & $\mathscr{A} \mid \mathscr{D} \varphi$ & $\mathrm{DR}, 1$ \\
\hline & $\varphi$ & $\mathscr{D E}, 2$ \\
\hline & $\mathscr{A} \varphi$ & $\mathscr{A} \mathrm{I}, 3$ \\
\hline & $\mathscr{D} \varphi \supset \mathscr{A} \varphi$ & $\supset \mathrm{I}, 1-4$ \\
\hline
\end{tabular}




\section{Concluding Remarks}

We have shown a sound and complete Fitch-style natural deduction system for an S5 modal logic endowed with diagonal operators and an actuality operator in a twodimensional semantics. The derivation rules presented here are analogous to Fitch's own modal rules for the one-dimensional case, thereby preserving familiar features of this kind of natural deduction system. The procedure is somewhat straightforward once new labeled lines such as $\mathscr{A}$ and $\delta$ are introduced alongside the usual $\square$ labeled line. This is enough to make it possible to evaluate formulas with respect to a pair of worlds as dictated by the two-dimensional character of the system.

Acknowledgements Thanks to Alexander Kocurek, Andrew Parisi, Shawn Standefer, the UC Davis Llemma group, and two anonymous reviewers for comments on earlier drafts that greatly improved this paper.

\section{References}

Chalmers, D (1996) The Conscious Mind: In Search of a Fundamental Theory. Oxford University Press.

Chalmers, D (2004) Epistemic Two-Dimensional Semantics. Philosophical Studies, $118,153-226$.

Crossley, JN and Humberstone, L (1977) The logic of "actually". Reports on Mathematical Logic 8, 11-29.

Davies, M and Humberstone, L (1980) Two notions of necessity. Philosophical Studies, 38(1), 1-30.

Evans, G (1979) Reference and Contingency. The Monist, 62, 161-189.

Fitch, FB (1952) Symbolic Logic: an Introduction. New York: Ronald Press, Co.

Fritz, P (2013) A logic for epistemic two-dimensional semantics. Synthese, 190, 1753-1770.

Fritz, P (2014) What is the correct logic of necessity, actuality, and apriority? The Review of Symbolic Logic, 7(3), 385-414.

Hazen, AP (1978) The eliminability of the actuality operator in propositional modal logic. Notre Dame Journal of Formal Logic, 4, 617-622.

Jackson F (1998a) From Metaphysics to Ethics: A Defense of Conceptual Analysis. Oxford University Press.

Kaplan, D (1989) Demonstratives. In Almog J, Perry J and Wettstein H (Eds.), Themes from Kaplan (pp. 481-563). Oxford: Oxford University Press.

Kripke, S (1963) Semantical analysis of modal logic I. Normal propositional calculi. Zeitschrift fur mathematische Logik und Grundlagen der Mathematik, 9(5-6):6796.

Kripke, S (1980) Naming and necessity. Cambridge, MA: Harvard University Press. Lampert, F (forthcoming) Actuality, tableaux, and two-dimensional modal logics. Erkenntnis. 
Restall, G (2012) A cut-free sequent system for two-dimensional modal logic, and why it matters. Annals of Pure and Applied Logic, 163, 1611-1623.

Stalnaker, RC (1978) Assertion. In Cole P (Ed.), Syntax and Semantics: Pragmatics (Vol. 9, pp. 315-339). New York: Academic Press. 